\title{
Micromanipulation, FIB, STEM, EDS and EELS of UF 4 Particles
}

\author{
Lucille A. Giannuzzi ${ }^{1,2^{*}}$, Michael DeVore $\mathrm{II}^{3}$, Michael Summer ${ }^{3}$ and Matthew Wellons ${ }^{3}$
}

1. L.A. Giannuzzi \& Associates LLC, Fort Myers, FL USA.

2. EXpressLO LLC, Lehigh Acres, FL USA.

3. Savanah River National Laboratory, Aiken, SC USA.

*Corresponding author: Lucille.Giannuzzi@EXpressLO.com

A key challenge with nuclear safeguards environmental sampling is chemical and structure characterization of particulate material post release due to environmentally induced chemical changes [1]. Uranium tetrafluoride $\left(\mathrm{UF}_{4}\right)$ is an important intermediate in the upstream and downstream chemical conversion process of uranium feedstock and metal production processes used in nuclear fuel production; however, minimal published research exists relating to $\mathrm{UF}_{4}$ hydrolysis [2]. Our effort utilized controlled environmental exposure to probe the induced chemical changes in $\mathrm{UF}_{4}$ test material and identified/characterized progeny species. However, initial characterization of the hydrolysis dynamics via Raman spectroscopy and SEM demonstrated that these methods did not adequately probe the observed material transformations demonstrated by the chemical and morphological changes (see figure 1). Advanced microanalytical methods were conducted to probe/characterize the internal material structure and chemical bonding via FIB, STEM, EDS, and EELS to cross-section and analyze the particle interiors.

The characterization of $U$ chemical states via EELS is an emergent field but recent efforts (including this one) demonstrate its feasibility [3]. The technical challenges include both; difficulty in preparing suitably thin lamella of uranium oxide specimens and a derth of publish reference information for U EELS absorption edges. Figure 2 shows the particle manipulation process performed on an EXpressLO Nicola ex situ lift out and micromanipulation station. A particle was identified and manipulated with a clean glass probe to a slotted EXpressLO ${ }^{\mathrm{TM}}$ grid that was previously painted with MBond glue [4]. The grid was moved to a desiccator evacuated to $\sim 10^{-2}-10^{-3} \mathrm{mbar}$ where the glue cured over time. FIB thinning was done on an FEI Helios NanoLab 660 with a final $2 \mathrm{keV} \mathrm{Ga}^{+}$ion polish. STEM and analytical work was performed on an FEI Titan ${ }^{3}$ operating at $200 \mathrm{keV}$.

Figure 3a shows an HAADF STEM image of small particle consisting of mostly equiaxed $\sim 200-500 \mathrm{~nm}$ grains. The particle shows porosity throughout. STEM/EDS mapping of the (a) entire particle and (b) its edge demonstrates O-rich and F-deficient sites that coincide with the exposed material surface, or internal pores that have formed due humidity exposure. STEM/EELS was able to chemically characterize the U, $\mathrm{O}, \mathrm{F}$ present within the multiple layers near the material surface, and internal pores show reduced $\mathrm{F}$ content relative to the bulk material (figure 4). Layers $500 \mathrm{~nm}$ from the material surface contain elevated O content consistent with expected hydrolysis products and the formation of an oxide coating or surface. The internal and surface deficiencies are consistent with prior Raman spectroscopy characterization where the prolonged hydrolysis of $\mathrm{UF}_{4}$ resulted in the formation of $\mathrm{U}^{6+}$ species $\left(\mathrm{UO}_{\mathrm{x}} \mathrm{F}_{\mathrm{y}}\right)$ via oxidation with reduced F content [2].

References:

[1] R. Kips et al., Proc. Radiochim. Acta, 1 (2011) p. 7-11.

[2] M. DeVore et al., SRNL 2016 LDRD External Report, (2017), SRNL-MS-2017-00048 p. 101

[3] J. Darnbrough et al., J. of Nuc. Mat. Volume, 502 (2018) p. 9-19.

[4] S. Vitale et al., Microsc. Microanal., 22 (2016) p. 80. 


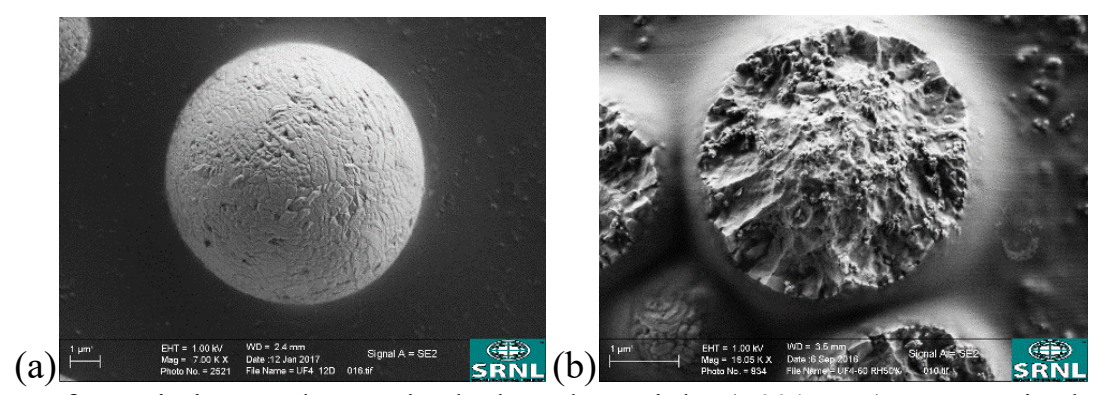

Figure 1. SEM image of a pristine and post hydrolyzed particle $(50 \% \mathrm{RH})$, respectively, which demonstrate characteristic surface morphology changes due to hydrolysis and mass transport.

(a)

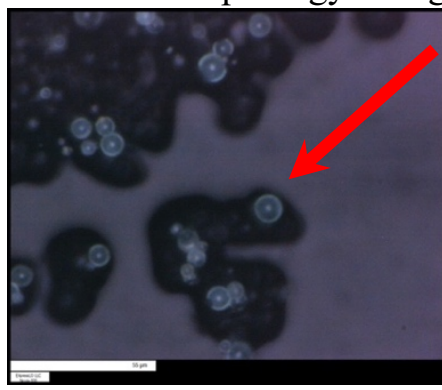

(b)

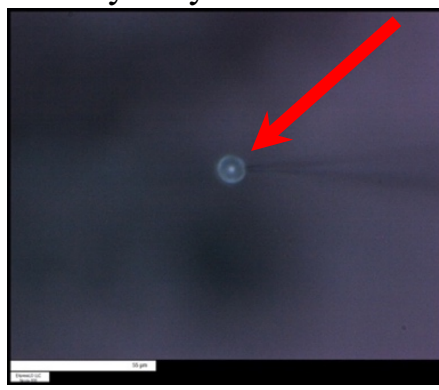

(c)

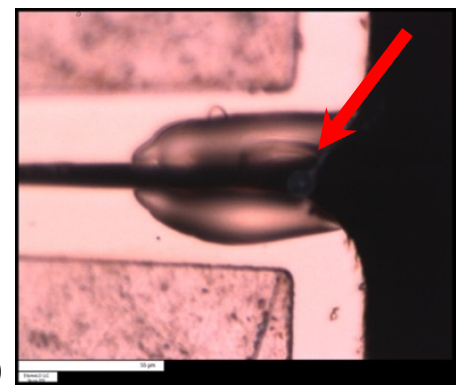

Figure 2. (a) particle identification, (b) particle removal via micromanipulation, (c) particle manipulated to glue on EXpressLO $^{\mathrm{TM}}$ slotted grid.

(a)
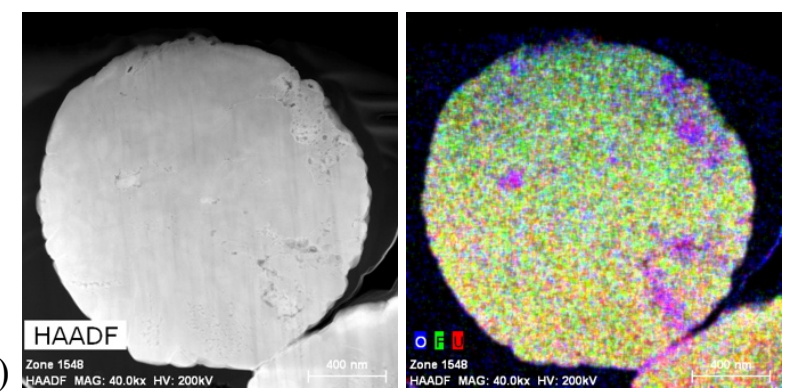

(b)

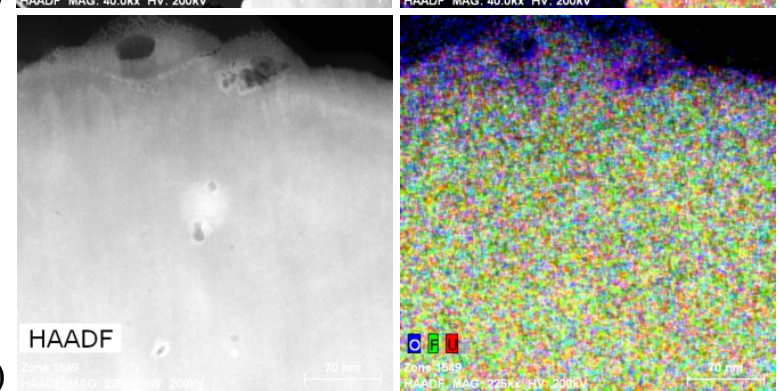

Figure 3. STEM images with O, F, U EDS map overlays from (a) an entire small particle and (b) the particle edge.
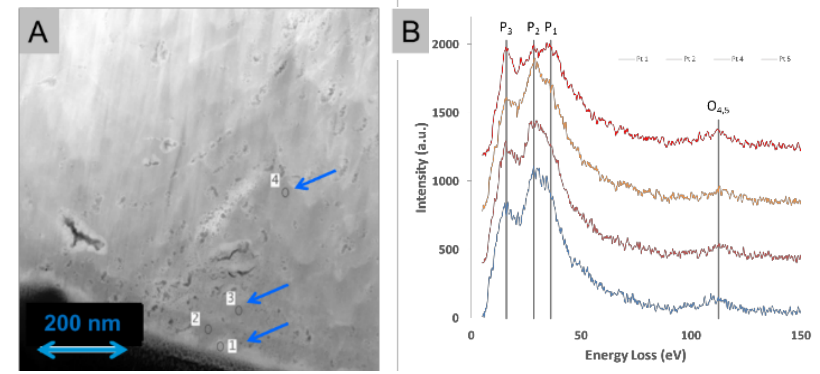

Figure 4. (a) HAADF STEM image (b) EELS spectra collected near the surface at position 1-4. The low loss regions show the $\mathrm{U}$ absorption edge peaks for $\mathrm{P}$ and O-type electronic transition states. 